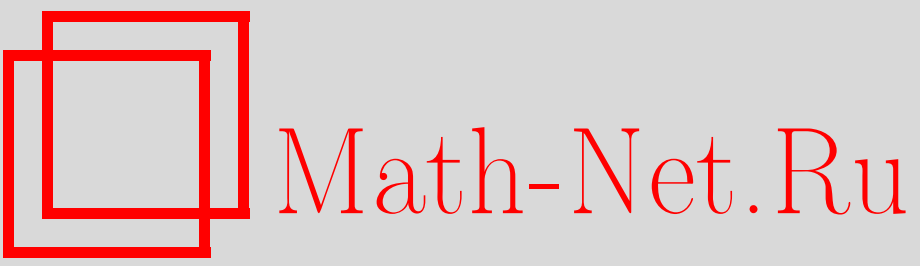

В. Е. Слюсарчук, Условия существования ограниченных решений нелинейных дифференциальных уравнений, УМН, 1999, том 54, выпуск 4, 181-182

DOI: https://doi.org/10.4213/rm193

Использование Общероссийского математического портала Math-Net.Ru подразумевает, что вы прочитали и согласны с пользовательским соглашением

http://www.mathnet.ru/rus/agreement

Параметры загрузки:

IP: 44.207 .124 .84

26 апреля 2023 г., 16:10:39 


\title{
УСЛОВИЯ СУЩЕСТВОВАНИЯ ОГРАНИЧЕННЫХ РЕШЕНИЙ НЕЛИНЕЙНЫХ ДИФФЕРЕНЦИАЛЬНЫХ УРАВНЕНИЙ
}

\author{
В. Е. СЛЮСАРЧУК
}

Пусть $f: \mathbb{R} \rightarrow \mathbb{R}$ - непрерывное отображение, $R(f)=\{f(x): x \in \mathbb{R}\}$ и $C^{0}$ - банахово пространство непрерывных и ограниченных на $\mathbb{R}$ функций $x=x(t)$ со значениями в $\mathbb{R}$ с нормой $\|x\|=\sup _{t \in \mathbb{R}}|x(t)|$.

Укажем необходимые и достаточные условия существования ограниченных решений дифференциального уравнения

$$
\frac{d x}{d t}=f(x)-h(t), \quad t \in \mathbb{R}
$$

с произвольной функцией $h=h(t) \in C^{0}$.

Теорема. Уравнение (1) для каждой функции $h=h(t) \in C^{0}$ имеет по крайней мере одно решение $x=x(t) \in C^{0}$ тогда и только тогда, когда $R(f)=\mathbb{R}$. При этом для каждого отрезка $[\alpha, \beta]$, для которого $R\left(\left.f\right|_{[\alpha, \beta]}\right)=\overline{R(h)} u\{f(\alpha), f(\beta)\}=$ $\left\{\inf _{t \in \mathbb{R}} h(t), \sup _{t \in \mathbb{R}} h(t)\right\}$, найдется решение $x=x(t) \in C^{0}$ maкое, что $R(x) \subset[\alpha, \beta]$.

Доказательство теоремы основывается на следующих леммах.

Обозначим через $\mathscr{F}$ класс всех непрерывных отображений $g: \mathbb{R} \rightarrow \mathbb{R}$, для каждого из которых $R(g)=\mathbb{R}$ и $\lim _{|x| \rightarrow+\infty}|g(x)|=+\infty$, а через $\mathscr{P}_{T}$ (где $T$ - положительное число) подпространство пространства $C^{0}$, состоящее из $T$-периодических функций.

Лемма 1. Пусть $f \in \mathscr{F}$. Тогда уравнение (1) для каждой функции $h=h(t) \in \mathscr{P}_{T}$ имеет по крайней мере одно решение $x=x(t) \in \mathscr{P}_{T}$.

ДокАЗАТЕЛЬСтво. Возьмем такие числа $k \neq 0$ и $a>0$, чтобы

$$
|f(x)-k x| \leqslant|k| a-\|h\|, \text { если }|x| \leqslant a,
$$

и рассмотрим вполне непрерывное отображение $G: \mathscr{P}_{T} \rightarrow \mathscr{P}_{T}$, определенное равенством

$$
(G x)(t)=-\frac{k}{|k|} \int_{\{s: k t<k s\}} e^{k(t-s)}(f(x(s))-k x(s)-h(s)) d s, \quad t \in \mathbb{R} .
$$

Нетрудно убедиться в том, что задача о сушествовании $T$-периодических решений уравнения (1) равносильна аналогичной задаче для уравнения

$$
x(t)=(G x)(t), \quad t \in \mathbb{R},
$$

на основании (2) $G S_{a} \subset S_{a}$, где $S_{a}=\left\{x \in \mathscr{P}_{T}:\|x\| \leqslant a\right\}$. Поэтому согласно теореме Шаудера о неподвижной точке $[1$, c. 36$]$ множество $T$-периодических решений уравнения $(3), \mathrm{a}$ следовательно, и уравнения (1) непусто.

Лемма 2. Пусть $f: \mathbb{R} \rightarrow \mathbb{R}$ - непрерывное отображсение, $h(t) \in \mathscr{P}_{T} u[a, b],[\alpha, \beta]$ - такие отрезки, что $R\left(\left.f\right|_{[\alpha, \beta]}\right)=R(h)=[a, b] u\{f(\alpha), f(\beta)\}=\{a, b\}$. Тогда уравнение (1) имеет решение $y(t) \in \mathscr{P}_{T}$, для которого $R(y) \subset[\alpha, \beta]$. 
ДокаЗАтельство. Рассмотрим отображение $f^{*} \in \mathscr{F}$, для которого $\left.f^{*}\right|_{[\alpha, \beta]}=\left.f\right|_{[\alpha, \beta]}$ и

$$
f^{*}(x) \in \mathbb{R} \backslash[a, b] \quad \forall x \in \mathbb{R} \backslash[\alpha, \beta] .
$$

Согласно лемме 1 уравнение

$$
\frac{d y}{d t}=f^{*}(y)-h(t), \quad t \in \mathbb{R},
$$

имеет решение $y(t) \in \mathscr{P}_{T}$, которое в некоторых точках $t_{1}$ и $t_{2}$ принимает наименьшее $y_{\min }$ и наибольшее $y_{\max }$ значения. В этих точках $d y / d t=0$. Поэтому $\left\{f^{*}\left(y_{\min }\right), f^{*}\left(y_{\max }\right)\right\}=$ $\left\{h\left(t_{1}\right), h\left(t_{2}\right)\right\}$ и на основании $(4)\left\{y_{\min }, y_{\max }\right\} \subset[\alpha, \beta]$. Следовательно, $R(y) \subset[\alpha, \beta]$. Поскольку $f^{*}(x)=f(x)$ для всех $x \in[\alpha, \beta]$, то решение $y=y(t)$ уравнения $(5)$ также является решением уравнения (1).

ДоКАЗАТЕЛЬСтво теОРемЫ. Пусть $R(f)=\mathbb{R}$. Рассмотрим последовательность чисел $T_{n}>0$ и функций $h_{n}(t) \in \mathscr{P}_{T_{n}}, n \in \mathbb{N}$, для которых $\lim _{n \rightarrow \infty} T_{n}=+\infty$,

$$
R\left(h_{n}\right)=\overline{R(h)}, \quad n \in \mathbb{N},
$$

$$
\lim _{n \rightarrow \infty} \max _{|t| \leqslant p}\left|h(t)-h_{n}(t)\right|=0 \quad \forall p>0,
$$

а также отрезок $[\alpha, \beta]$, для которого $R\left(\left.f\right|_{[\alpha, \beta]}\right)=\overline{R(h)}$ и $\{f(\alpha), f(\beta)\}=\left\{\inf _{t \in \mathbb{R}} h(t)\right.$, $\left.\sup _{t \in \mathbb{R}} h(t)\right\}$. Согласно лемме 2 и равенствам (6) найдутся функции $x_{n}(t) \in \mathscr{P}_{T_{n}}, n \in \mathbb{N}$, такие, что $R\left(x_{n}\right) \subset[\alpha, \beta], n \in \mathbb{N}$, и

$$
x_{n}(t)=x_{n}(0)+\int_{0}^{t}\left(f\left(x_{n}(s)\right)-h_{n}(s)\right) d s, \quad k \in \mathbb{N}, \quad t \in \mathbb{R} .
$$

Тогда $\sup \left\{\mid x_{n}^{\prime}(t): n \in \mathbb{N}, t \in \mathbb{R}\right\} \leqslant \max \{|a|, b\}+\|h\|$. Следовательно, функции $x_{n}(t), n \in \mathbb{N}$, не только равномерно ограничены, но и равностепенно непрерывны на $\mathbb{R}$. Поэтому на основании теоремы Арцела [2, с. 106] найдутся функция $z(t) \in C^{0}$ и числа $n_{k}>k, k \in \mathbb{N}$, для которых $R(z) \subset[\alpha, \beta]$ и

$$
\lim _{k \rightarrow \infty} \max _{|t| \leqslant p}\left|z(t)-x_{n_{k}}(t)\right|=0 \quad \forall p>0 .
$$

В силу (7)-(9) и непрерывности отображения $f$

$$
z(t)=z(0)+\int_{0}^{t}(f(z(s))-h(s)) d s, \quad t \in \mathbb{R},
$$

т.е. $z(t)$ - решение уравнения (1).

Пусть $R(f) \neq \mathbb{R}$. Рассмотрим уравнение

$$
\frac{d x}{d t}=f(x)-m, \quad t \in \mathbb{R},
$$

где $m \in \mathbb{R} \backslash \overline{R(f)}$. Каждое решение $x(t)$ этого уравнения является неограниченным, поскольку $\left|x^{\prime}(t)\right| \geqslant \inf \{|m-y|: y \in R(f)\}>0$ для всех точек $t$ из множества определения этого решения. Отсюда следует, что существование ограниченных решений уравнения (1) с произвольной функцией $h(t) \in C^{0}$ обеспечивает выполнение соотношения $R(f)=\mathbb{R}$, что завершает доказательство теоремы.

\section{СПИСОК ЛИТЕРАТУРЫ}

[1] Ниренберг Л. Лекции по нелинейному функциональному анализу. М.: Мир, 1977. [2] Колмогоров А. Н., Фомин С. В. Элементы теории функций и функционального анализа. М.: Наука, 1968. 